\title{
1 Noncontractile tissue forces mask muscle fiber forces underlying muscle 2 spindle Ia afferent firing rates in stretch of relaxed rat muscle
}

4 Kyle P. Blum ${ }^{1}$, Paul Nardelli ${ }^{2}$, Timothy C. Cope ${ }^{1,2,3}$, Lena H. Ting ${ }^{1,3}$

$5 \quad{ }^{1}$ Wallace H. Coulter Dept. of Biomedical Engineering, Georgia Institute of Technology

$6 \quad{ }^{2}$ School of Biological Sciences, Georgia Institute of Technology

$7 \quad{ }^{3}$ Dept. of Rehabilitation Medicine, Division of Physical Therapy, Emory University

9 Abstract

10 Stretches of relaxed cat and rat muscle elicit similar history-dependent muscle spindle Ia firing rates that

11 resemble history-dependent forces seen in single activated muscle fibers (Nichols and Cope, 2004). During

12 stretch of relaxed cat muscle, whole musculotendon forces exhibit history-dependence that mirror history-

13 dependent muscle spindle firing rates, where both muscle force and muscle spindle firing rates are elevated

14 in the first stretch in a series of stretch-shorten cycles (Blum et al., 2017). By contrast, rat musculotendon

15 are only mildly history-dependent and do not mirror history-dependent muscle spindle firing rates in the

16 same way (Haftel et al., 2004). We hypothesized that history-dependent muscle spindle firing rates elicited

17 in stretch of relaxed rat muscle would mirror history-dependent muscle fiber forces, which are masked by

18 noncontractile tissue at the level of whole musculotendon force. We removed noncontractile tissue force

19 contributions from the recorded musculotendon force using an exponentially-elastic tissue model. We then

20 show that the remaining estimated muscle fiber force resembles history-dependent muscle spindle firing

21 rates recorded simultaneously. These forces also resemble history-dependent forces recorded in stretch of

22 single activated fibers and attributed to muscle cross-bridge mechanisms (Campbell and Moss, 2000). Our

23 results suggest that history-dependent muscle spindle firing in both rats and cats arise from stretch of cross-

24 bridges in muscle fibers. 


\section{Introduction}

Muscle spindles are sensory organs within skeletal muscles that are crucial for sensing body segment position and motion (Prochazka and Ellaway, 2012) with mechanosensory signaling characteristics that generalize across species (Vincent et al., 2017). In relaxed muscle, muscle spindle Ia afferents fire when muscles are stretched by an external load, beginning with a high-frequency initial burst of firing,

\section{2} followed by increased firing related to stretch velocity and amplitude in cats, rats, rabbits, and humans (Blum et al., 2017; Proske and Stuart, 1985; Vincent et al., 2017). However, these responses are history

34 dependent, such that the first stretch in a series of identical stretch-shorten cycle elicits an initial burst and 35 response to ramp stretches that are absent or reduced in subsequent stretches (Blum et al., 2017; Haftel et al., 2004; Matthews, 1972; Proske and Stuart, 1985).

In anesthetized cats, history-dependent muscle spindle firing rates mirror history-dependent whole musculotendon forces during muscle stretch. The fine temporal details of Ia afferent firing rates can be precisely reproduced through weighted pseudolinear combinations of recorded whole musculotendon force and $\mathrm{dF} / \mathrm{dt}$ signals (Blum et al., 2017). As such, elevated whole musculotendon force and $\mathrm{dF} / \mathrm{dt}$ in the first

41 stretch directly explain the initial bursts and elevated firing rates in the first stretch in a series of stretch42 shorten cycles.

In anesthetized rats, however, the history-dependent muscle spindle firing rates cannot be directly

44 explained by history-dependence in whole musculotendon forces (Haftel et al., 2004). Specifically, the

45 force profiles are qualitatively different the muscle spindle firing rates and the forces observed in cat 46 muscle, exhibiting an exponential increase in force during ramp stretches. As longer strain was elicited in 47 prior rat vs cat studies $\left(7 \%\right.$ vs. $\left.3 \% \mathrm{~L}_{0}\right)$ greater non-contractile tissues are likely engaged underlying the 48 exponential rise in musculotendon force during stretch (Meyer and Lieber, 2011).

Here we hypothesized that history-dependent muscle fiber forces are masked by noncontractile 50 tissue forces when examining whole musculotendon force during muscle stretch in anesthetized rats. We

51 further hypothesized that muscle fiber forces in rats exhibit similar history-dependence as muscle spindle

52 Ia firing rates recorded simultaneously. We made the simplifying assumption that noncontractile tissue 
53 force and muscle fiber force act in parallel, contributing additively to whole musculotendon force.

54 Noncontractile forces were estimated using a simple exponential tissue model, and muscle fiber force was

55 estimated by analytically removing noncontractile tissue force from recorded musculotendon force. We

56 found that a large proportion for whole musculotendon force to be carried by noncontractile tissues. Further,

57 the estimated muscle fiber force and its first time derivative, $\mathrm{dF} / \mathrm{dt}$, was history-dependent and closely

58 resembled history-dependent muscle spindle firing rates recorded simultaneously. Our work suggests that

59 the same cross-bridge mechanisms underlie history-dependent muscle spindle firing and muscle fiber forces

60 in rats and cats.

61

\section{Methods}

63 Animal care

64 All procedures and experiments were approved by the Georgia Institute of Technology's

65 Institutional Animal Care and Use Committee. Adult female Wistar rats ( $\mathrm{N}=5 ; 250$ - $300 \mathrm{~g})$ were studied

66 in terminal experiments only and were not subject to any other experimental procedures. All animals were

67 housed in clean cages and provided food and water ad libitum in a temperature- and light-controlled

68 environment in Georgia Institute of Technology's Animal Facility.

70 Terminal physiological experiments

71 Experiments were designed to measure the firing of individual muscle afferents in response to

72 muscle stretch in vivo using electrophysiological techniques as documented previously (e.g. (Vincent et al.,

73 2017)). Briefly described, rats were deeply anesthetized (complete absence of withdrawal reflex) by

74 inhalation of isoflurane, initially in an induction chamber $\left(5 \%\right.$ in $\left.100 \% \mathrm{O}_{2}\right)$ and, for the remainder of the

75 experiment, via a tracheal cannula $\left(1.5-2.5 \%\right.$ in $\left.100 \% \mathrm{O}_{2}\right)$.

76 The triceps-surae muscle group (medial and lateral gastrocnemius and soleus muscles) in the left

77 hindlimb was dissected free of surrounding tissue and detached at its insertion together with a piece

78 calcaneus bone. The severed insertion of the left triceps-surae muscle group and securely attached directly 
79 to the lever arm of a force and length-sensing servomotor (Model 305B-LR, Aurora Scientific Inc.), which

80 provided for controlled muscle stretch while recording muscle length and force (dual-mode lever arm

81 system, Aurora Scientific). Initial muscle tension was set to $0.1 \mathrm{~N}$, which is the approximate passive tension

82 observed for ankle and knee angles of $90^{\circ}$ and $120^{\circ}$, respectively.

83 Triceps surae sensory axons were randomly sampled by intra-axonal penetration in dorsal rootlets

84 and selected for detailed study when identified as group Ia based on several criteria (Vincent et al., 2017).

85 First, selected afferents were identified as low threshold mechanoreceptors supplying triceps surae muscles

86 when electrical stimulation of triceps surae nerves generated orthodromic action potentials with conduction

87 delay $<2.5 \mathrm{~ms}$. Second, muscle spindle afferents were differentiated by their response to electrically-evoked

88 isometric twitch contractions, which resulted in cessation of stretch-evoked firing in distinction with group

89 Ib afferents which accelerated firing. Finally, group Ia afferents were distinguished from muscle-spindle

90 group II afferents when responded with high-frequency firing $(>100 \mathrm{~Hz})$ upon stretch of resting muscle and

91 with high fidelity firing at $100 \mathrm{~Hz}$ during triceps surae tendon vibration at $100 \mathrm{~Hz}$.

92 Firing rates and patterns of Ia afferents were studied in response to length-servo controlled stretches

93 applied to triceps surae muscles at rest, i.e. not engaged in active contraction. Triangular stretches, i.e.

94 ramp-release stretch at constant velocity $(4 \mathrm{~mm} / \mathrm{s})$ were applied with amplitudes ranging from 0.5 to $3 \mathrm{~mm}$.

95 Trials of sequential 3-5 stretches were repeated in between rest periods, i.e. muscles held at resting length

96 for at least 10 seconds allowing expression of history dependent firing responses (Fig 1B; (Haftel et al.,

97 2004)). Whole-muscle force, length changes, and Ia-afferent action potentials were simultaneously

98 recorded at a sampling rate of at least $20 \mathrm{kHz}$ and down-sampled to at least $1 \mathrm{kHz}$ for analysis. Yank was

99 calculated as the numerical derivative of recorded force. Ia-afferent action potential discriminated for off-

100 line analysis of firing rates and patterns.

101

102

\section{Muscle fiber force estimation}



noncontractile tissues arranged in parallel muscle fibers (Fig. 1C). We assumed noncontractile tissues were purely elastic with both exponential and linear stiffness (Fig. 1D) describe by the following equation:

$$
F_{n c}=k_{l i n}\left(L-L_{0}\right)+A e^{k_{\exp }\left(L-L_{0}\right)}
$$

Where $\mathrm{L}$ is the entire musculotendon length, $\mathrm{L}_{0}$ is the musculotendon resting length, and $k_{\text {lin }}, A$, and $k_{\exp }$ spring constants for linear and exponential elements, respectively. Noncontractile tissue forces were then subtracted from the recorded force to estimate the muscle fiber force, which resembled muscle spindle IFRs amount of variance of the recorded Ia afferent IFR during $2 \mathrm{~mm}$ or $3 \mathrm{~mm}$ stretch trials (Blum et al., 2017). force in all stretches of that muscle.

Variance accounted for of musculotendon force by estimated noncontractile force

where the numerator represents the sum of squares of the noncontractile tissue model for a given trial and

121 the denominator is the total sum of squares of the recorded musculotendon force for the same trial. 


$$
\widehat{I F R}=\left(F(t)+b_{F}\right) * k_{F}+\left(\frac{d F(t)}{d t}+b_{\frac{d F}{d t}}\right) * k_{\frac{d F}{d t}}+C
$$

$129 k_{F}$ and $k_{d F / d t}$, respectively, are constant weights on estimated fiber force and $\mathrm{dF} / \mathrm{dt}$ and $b_{F}$ and $b_{d F / d t}$,

130 respectively, are constant offsets on fiber force and dF/dt. $C$ is a constant offset. For visualization, we chose

131 these parameters to be $k_{F}=1393$ spikes $/ \mathrm{Ns}, k_{d F / d t}=3.6$ spikes $/ \mathrm{N}, b_{F}=10 \mathrm{~N}, b_{d F / d t}=5 \mathrm{~N} / \mathrm{s}$, and $C=34$

132 spikes/s. Additionally, the yank and force components were subjected to a force threshold to emulate the 133 spiking threshold of the neuron.

\section{Results and Discussion}

Muscle spindle firing rates appeared history-dependent in rats but muscultotendon force did not

As described previously, rat muscle spindle Ia afferents exhibited history-dependent firing rates in response to repeated ramp-release stretch perturbations (Haftel et al., 2004). Initial bursts were observed at 1B). In contrast, simultaneously recorded musculotendon force did not appear history-dependent, apart

141 from a small rise in force at the onset of stretch primarily visible in the yank time series (Fig. 1B) and 142 exhibited an exponentially increasing force with stretch length (Fig. 1C).

Estimated muscle fiber force exhibited history dependence analogous to that observed in muscle spindle 145 spike rates

Non-contractile force contributions to whole musculotendon force increased with stretch length

147 (Fig 1D). Both the whole musculotendon force and $\mathrm{dF} / \mathrm{dt}$ signal exhibited a similar nonlinear rise with 148 applied, a property of an exponential relationship (Fig 1D). The non-contractile component was thus 149 modeled by linear and exponential parameters of $k_{\text {lin }}=0.0497 \pm 0.0388, A=0.0454 \pm 0.317, k_{\text {lin }}=1.071$ $150 \pm 0.362$ (mean \pm sd.; $\mathrm{N}=5$ muscles). Using a single set of parameters for each muscle analyzed, the estimated 
noncontractile force component accounted for $53 \pm 3 \%$ (mean \pm S.E.M.) of the total variance in force for 3 $\mathrm{mm}$ stretches, $34 \pm 3 \%$ for $2 \mathrm{~mm}$ stretches, $12 \pm 1 \%$ for $1 \mathrm{~mm}$ stretches, and only $3 \%$ (based on data from one muscle) for $0.5 \mathrm{~mm}$ stretches.

The remaining forces represent the estimated muscle fiber force over time, which exhibited historydependence (Fig. 1E). The estimated muscle fiber force had a pronounced initial force rise and larger initial peak in the first stretch (Fig. 1E). This was accompanied by a larger peak in $\mathrm{dF} / \mathrm{dt}$ in the first stretch (Fig. 1E). Additionally, this rapid force increased resulting in a higher overall level of force during the first ramp stretch compared to subsequent stretches (Fig 1E).

The history-dependence of muscle spindle firing rates, whole musculotendon force and $\mathrm{dF} / \mathrm{dt}$, the and estimated muscle fiber force and $\mathrm{dF} / \mathrm{dt}$ are illustrated by differences in the first stretch response versus subsequent responses (defined in Fig. 2A). Muscle spindle firing rates were dramatically different on the first stretch compared to subsequent stretches, which were all similar to each other (Fig. 2B, compare yellow resembled the history-dependence of the muscle spindle firing rates.

We found that the history dependence seen in muscle spindle firing rates (Fig. 3A, B) resembled linear combinations of muscle fiber force and $\mathrm{dF} / \mathrm{dt}$, subject to a threshold (Fig. 3C, D). Although muscle

170 spindle firing rates in $0.5 \mathrm{~mm}$ stretches (e.g. Fig 3B) were qualitatively different than $2 \mathrm{~mm}$ stretches (Fig $1713 \mathrm{~A}$ ), they were still similar to the linear combinations of the estimated muscle fiber force and yank (Fig. C,

172 D). Morevoer, these estimates were generated using the same weighting of force and yank at both stretch

173 lengths (Fig. 3 E, F). This robustness is all the more remarkable because the same noncontractile tissue

174 properties were used to estimate muscle fiber force and $\mathrm{dF} / \mathrm{dt}(\mathrm{Fig} 3 \mathrm{G}, \mathrm{H})$ at different stretch lengths where

175 the amplitude of the noncontractile forces differed dramatically $(30.2 \%$ vs $3.1 \%$ VAF of the total 176 musculotendon force, respectively). In particular the flatter muscle spindle response during the first ramp- 
177 release (Fig. B, yellow trace) was also present in the linear combination of muscle fiber force and yank 178 (Fig. 3D, yellow trace).

In summary, we show in anesthetized rats that history-dependent muscle fiber forces during stretch of relaxed muscle can be identified from whole musculotendon force even when the majority of that force

181 arises from stretch of non-contractile tissues. Exponential non-contractile force dominated the 182 musculotendon force and yank traces, consistent with reports that muscle fibers carry as little as $15 \%$ of 183 total musculotendon loads in rodents ((Meyer and Lieber, 2011), Meyer and Lieber, in review). The 184 remaining residual force was a relatively small component of the total force but resembled characteristics 185 of isolated, activated, muscle fibers in ramp-release stretches (Campbell and Lakie, 1998; Campbell and 186 Moss, 2000; Campbell and Moss, 2002), with an initial short-range stiffness (Getz et al., 1998), as well as 187 higher mean force during the first ramp stretch.

188 Further, estimated muscle fiber forces exhibited history-dependent characteristics that were similar to history-dependence in muscle spindle afferents recorded simultaneously, suggesting similar muscle cross-bridge mechanisms in both cats and rats. These properties have also been shown to arise from musclecross bridge dynamics and only occur in the presence of $\mathrm{Ca}++$ (Campbell and Moss, 2002). The firing rates of muscle spindle primary afferents are directly related to the intrafusal muscle fibers of the spindle, with only a small amount of connective tissue force contributing to the spike-generating force (Boyd et al., 1977; Hunt and Ottoson, 1975; Hunt and Wilkinson, 1980). In relaxed muscle we assume that the intrafusal and extrafusal muscle force exhibit similar responses to stretch, and therefore used our overall estimate of muscle fiber force as a proxy for intrafusal fiber force. As such our work shows that both rat and cat muscle 197 spindles exhibit history-dependence that arises directly from muscle cross-bridge dynamics when stretched, 198 driving the firing behavior.

Our study further provides a neuromuscular explanation for the tendency of human subjects to 200 underestimate forces at longer muscle lengths due to the stretch of non-contractile tissues. Psychophysical 201 studies demonstrate that human participants tend to only perceive forces generated by muscle fibers, and 202 not passive components of force (Tsay et al., 2014). This sense of "fiber-only" force is consistent with the 
203 finding in the present study that muscle spindles only fire in response to the force in muscle fibers, but not

204 in non-contractile tissues. While Golgi tendon organs also sense active muscle force, they are located in-

205 series with both muscle fibers and passive connective tissues and are unable to differentiate contractile and

206 non-contractile force. The high contribution of non-contractile forces to whole musculotendon force during

207 muscle stretch in rats could also explain the fact that Golgi tendon organ Ib afferents fire robustly to stretch

208 in rats, but not cats (Vincent et al., 2017).

209 Taken together, our work suggests muscle spindle firing rate reflect muscle fiber forces during

210 passive stretch across different species. The dissociation of the encoding of contractile and non-contractile

211 forces may be important in understanding the role of proprioceptors in sensorimotor control, and the

212 similarities and differences across species (Vincent et al., 2017). Differences in prior experiments between

213 cats and rats may simply be due to difference in the relative amplitude of stretches, as larger stretches in

214 cat muscle do engage non-contractile force elements that have an exponential characteristic (Matthews,

215 1931) and would likely need to be considered for larger stretch amplitudes. Indeed, when homologous

216 muscles in the cat, rat, and mouse are stretched with similar strain, firing responses of Ia afferents in each

217 species are similar despite differences in the shape of musculotendon force (Carrasco et al., 2017). Further,

218 in our anesthetized conditions, intrafusal forces within the muscle spindle encoding region is assumed to

219 be similar to the extrafusal muscle force; however, intrafusal muscle force would be increased in conditions

220 where gamma motor neurons innervating intrafusal muscle fibers are active. However, we hypothesize that

221 the fundamental idea that muscle spindles fire in response to intrafusal muscle force and yank will

222 generalize across both passive and active movement conditions. 
A

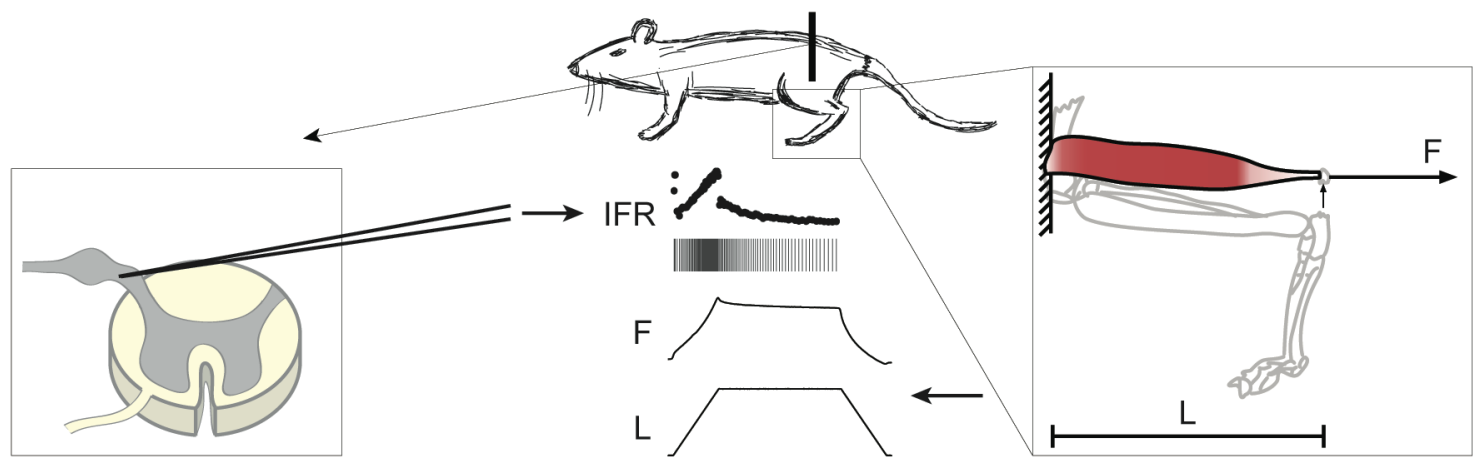

B

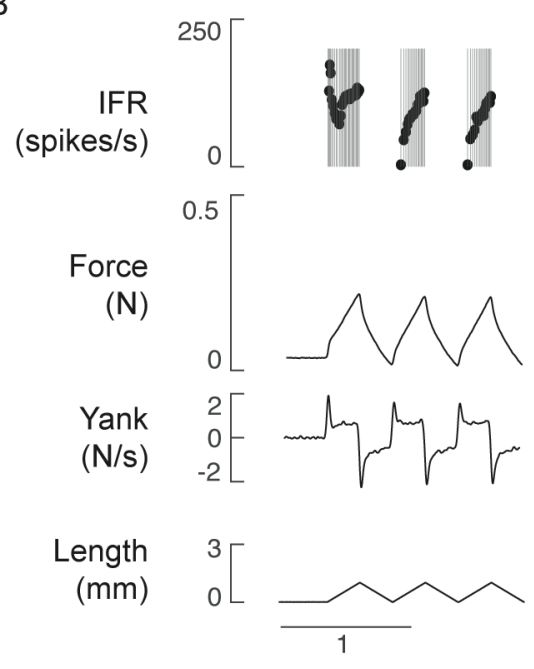

C

$$
F_{\text {Fiber }}=F_{M T}-F_{\text {noncontractile }}
$$

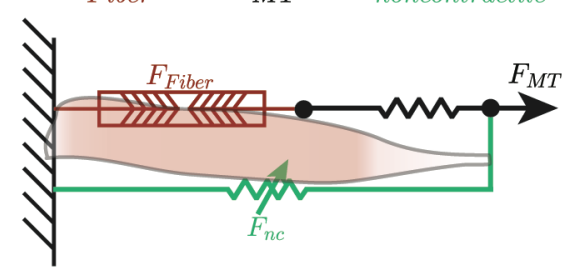

$\underline{1 \mathrm{~s}}$

D $\quad$ Length $2 \mathrm{~mm} /$

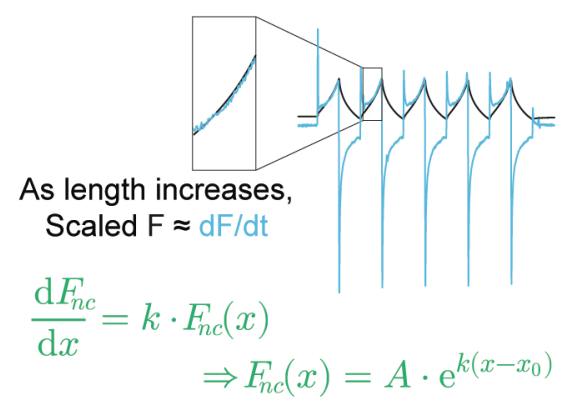

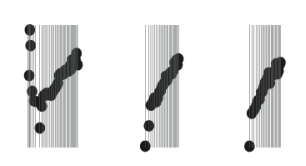
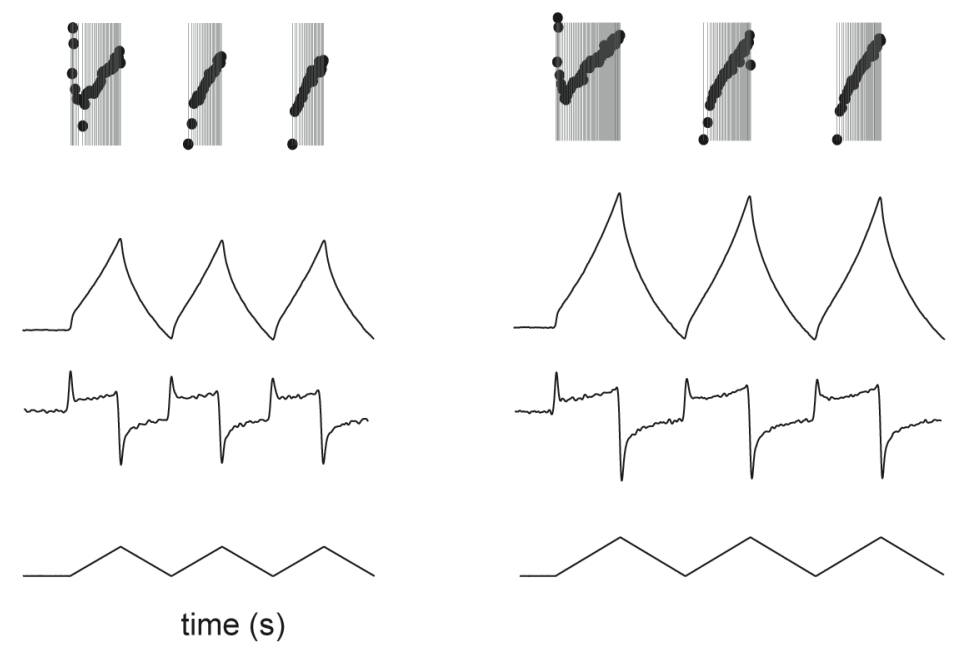

$\mathrm{E}$

$0.5 \mathrm{~N}$
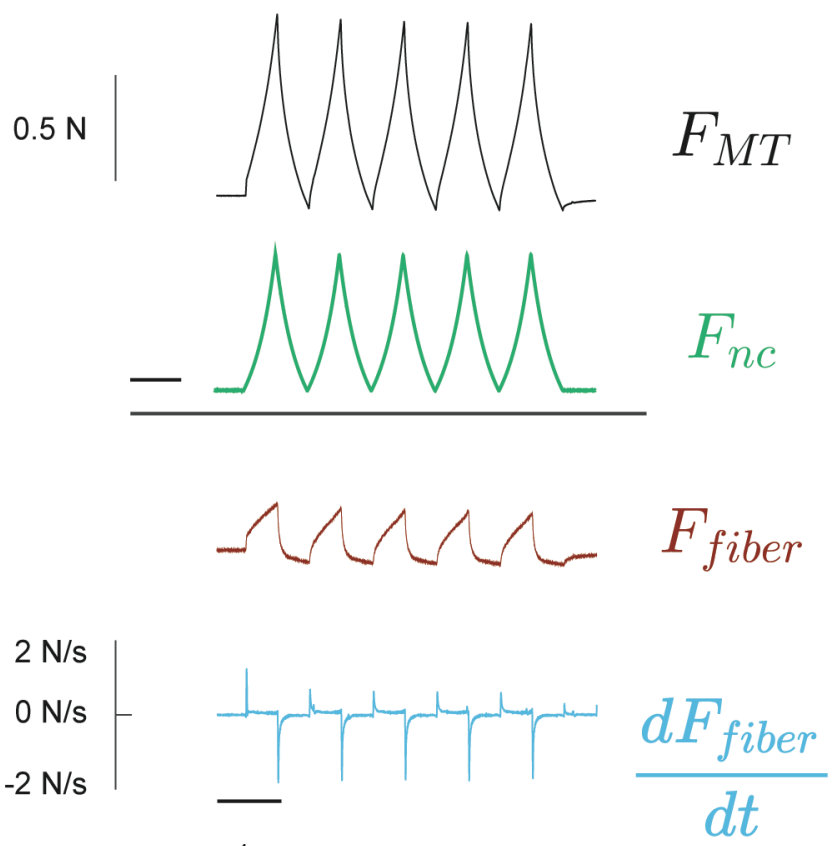

$1 \mathrm{~s}$ 
227 Figure 1: Methods for recording muscle spindle firing rates and estimating muscle fiber force during stretch

228 of relaxed rat muscle. A) Intra-axonal recording from muscle spindle Ia afferents from in dorsal rootlet

229 were recorded during stretch of the triceps surae musculotendon. Muscle length (L) and total

230 musculotendon force (F; panel on right) were recorded with membrane potentials; instantaneous firing rate

231 were computed based on action potential events (IFR; traces in center panel). B) Sets of identical triangular

232 stretches were imposed at three lengths $(1 \mathrm{~mm}, 2 \mathrm{~mm}$, and 3mm). Action potentials (gray lines) with

233 superimposed instantaneous firing rate (black dots) are shown temporally aligned with simultaneously

234 recorded muscle force, yank and length traces. In each trial, muscle spindle Ia firing response to identical

235 stretch stimuli different in the first stretch when compared with subsequent stretches. Specifically, the

236 response to the first stretch contains an initial burst of spikes at stretch onset and more pronounced dynamic

237 response post-initial burst. C) The recorded musculotendon force was assumed to be comprised two additive

238 force components: one component arising from noncontractile tissues (green) with an elastic force-length

239 relationship and another component arising from the muscle fibers (red) and series elastic component

240 (black). D) The recorded musculotendon force (black) and calculated $\mathrm{dF} / \mathrm{dt}$ (cyan) signals exhibited an

241 exponential force-length relationship which was more dominant at longer stretch lengths, justifying the

242 model of noncontractile tissue as an exponential spring. E) Subtracting the estimated noncontractile force

243 component (green) from the recorded musculotendon force (black) yielded the estimated muscle fiber force

244 (red) and dF/dt (blue), both of which were history dependent, with a larger response during the first stretch. 
A

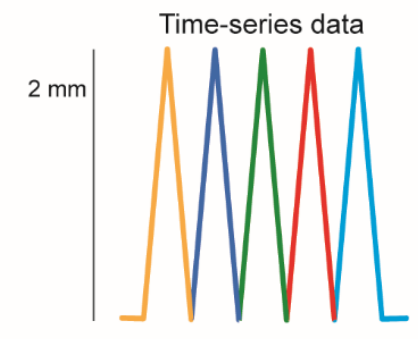

C

Recorded musculotendon force response
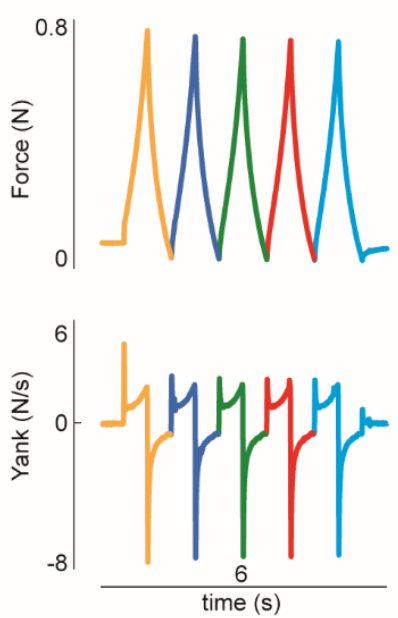

Hysteresis

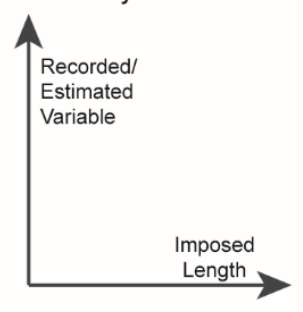

D
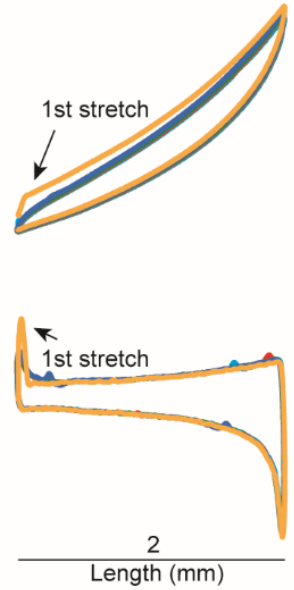

B

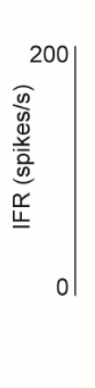

Recorded muscle spindle la spiking response
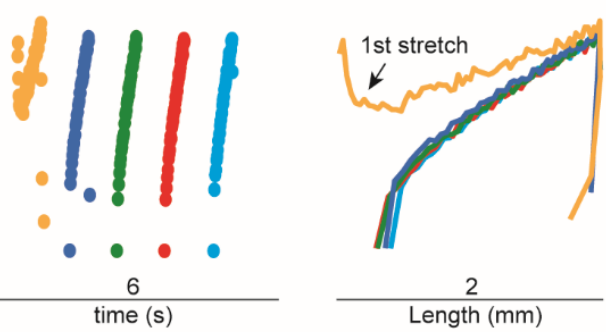

Estimated muscle fiber force response
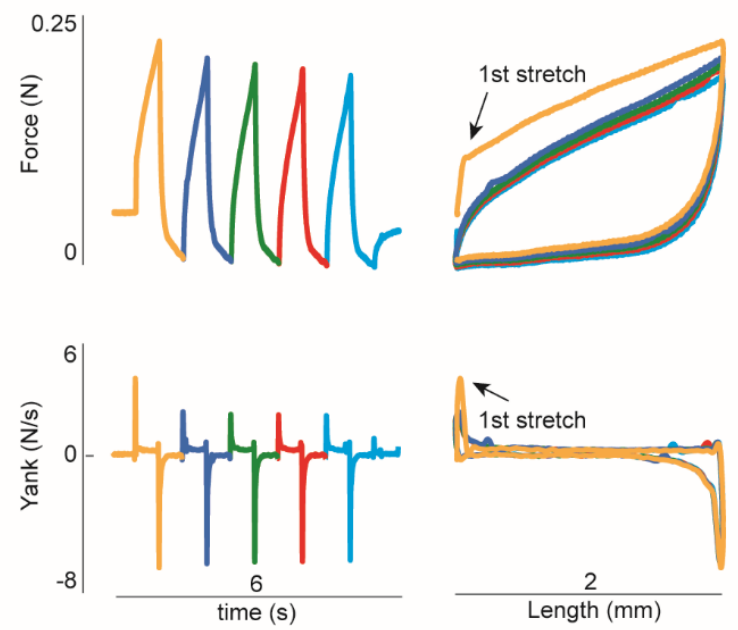

Figure 2: History-dependence of muscle spindle firing rates, musculotendon force and $\mathrm{dF} / \mathrm{dt}$, and muscle

247 fiber force and $\mathrm{dF} / \mathrm{dt}$. A) Applied length depicted as time series (left panel). Different colors represent

248 different stages of the perturbation. B) Recorded firing rate from Ia afferent to applied length from A.

249 Represented as time series (left) and as a function of length (right). C) Recorded musculotendon force (top)

250 and $\mathrm{dF} / \mathrm{dt}$ (bottom) D) Estimated muscle fiber force (top) and $\mathrm{dF} / \mathrm{dt}$ (bottom) estimated from musculotendon

251 force in $\mathrm{C}$. 
A

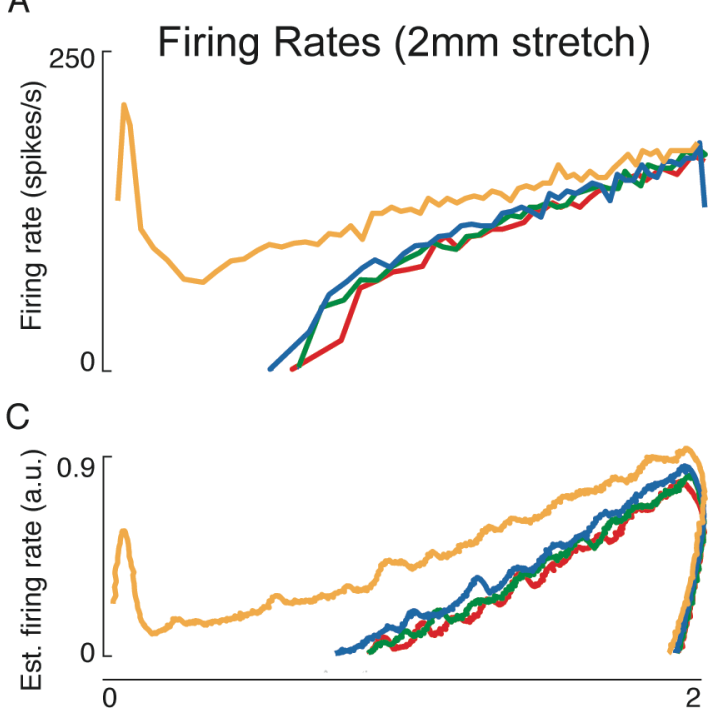

E

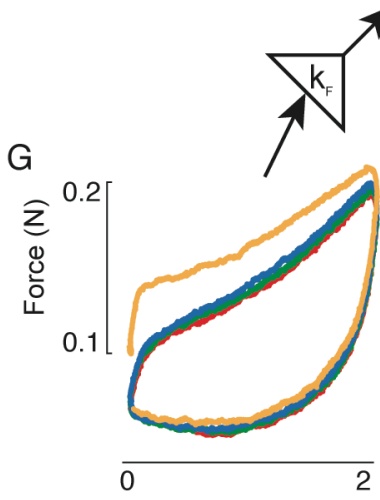

$\Sigma$

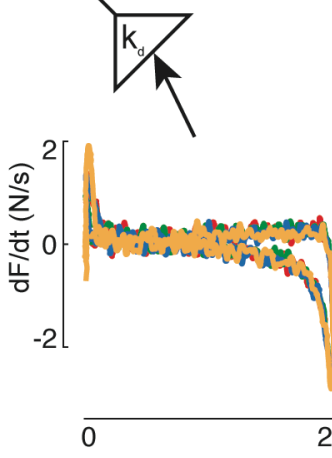

$\mathrm{B}$

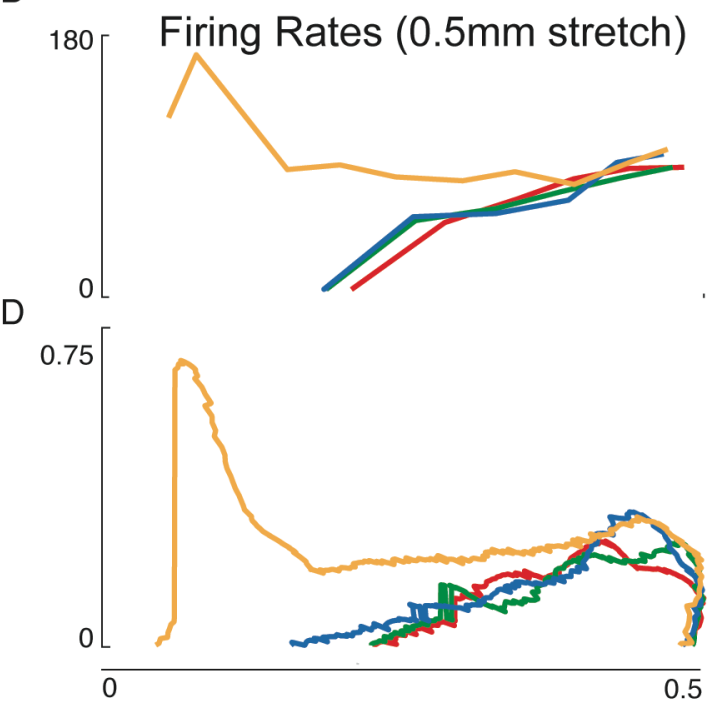

$\mathrm{F}$

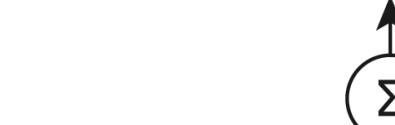

$\Sigma$

$\mathrm{H}$

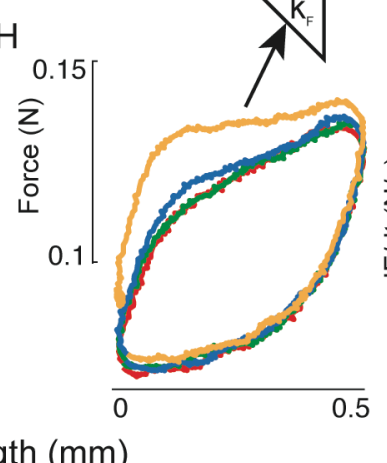

Length $(\mathrm{mm})$

252 Figure 3: History-dependent muscle spindle firing rates at different applied lengths resembled linear

253 combinations of muscle fiber force and $\mathrm{dF} / \mathrm{dt}$. Muscle spindle firing rate for the afferent in A) $2 \mathrm{~mm}$ and B)

$2540.5 \mathrm{~mm}$ stretches. C, D)Linear combination of estimated muscle fiber force and $\mathrm{dF} / \mathrm{dt}$ subject to a threshold

255 exhibited the same qualitative features in both stretch conditions. E,F) The same weights were used in both

256 stretch amplitudes to combine $\mathrm{G}, \mathrm{H}$ ) estimated muscle fiber force and $\mathrm{dF} / \mathrm{dt}$ signals. 


\section{References Cited}

Blum, K. P., Lamotte D'Incamps, B., Zytnicki, D. and Ting, L. H. (2017). Force encoding in muscle spindles during stretch of passive muscle. PLoS Comput. Biol. 13, e1005767-24.

Boyd, I. A., Gladden, M. H., McWilliam, P. N. and Ward, J. (1977). Control of dynamic and static nuclear bag fibres and nuclearbag fibres and nuclear chain fibres by gamma and beta axons in isolated cat muscle spindles. J. Physiol. (Lond.).

Campbell, K. S. and Lakie, M. (1998). A cross-bridge mechanism can explain the thixotropic shortrange elastic component of relaxed frog skeletal muscle. J. Physiol. (Lond.) 510 ( Pt 3), 941-962.

Campbell, K. S. and Moss, R. L. (2000). A thixotropic effect in contracting rabbit psoas muscle: prior movement reduces the initial tension response to stretch. J. Physiol. (Lond.) 525 Pt 2, 531-548.

Campbell, K. S. and Moss, R. L. (2002). History-dependent mechanical properties of permeabilized rat soleus muscle fibers. Biophysical Journal 82, 929-943.

Carrasco, D. I., Vincent, J. A. and Cope, T. C. (2017). Distribution of TTX-sensitive voltage-gated sodium channels in primary sensory endings of mammalian muscle spindles. Journal of Neurophysiology 117, 1690-1701.

Getz, E. B., Cooke, R. and Lehman, S. L. (1998). Phase transition in force during ramp stretches of skeletal muscle. Biophysical Journal 75, 2971-2983.

Haftel, V. K., Bichler, E. K., Nichols, T. R., Pinter, M. J. and Cope, T. C. (2004). Movement reduces the dynamic response of muscle spindle afferents and motoneuron synaptic potentials in rat. Journal of Neurophysiology 91, 2164-2171.

Hunt, C. C. and Ottoson, D. (1975). Impulse activity and receptor potential of primary and secondary endings of isolated mammalian muscle spindles. 252, 259-281.

Hunt, C. C. and Wilkinson, R. S. (1980). An analysis of receptor potential and tension of isolated cat muscle spindles in response to sinusoidal stretch. $J$. Physiol. (Lond.) 302, 241-262.

Matthews, B. H. C. (1931). The response of a muscle spindle during active contraction of a muscle. The Journal of Physiology 72, 153-174.

Matthews, P. B. C. (1972). Mammalian muscle receptors and their central actions.

Meyer, G. A. and Lieber, R. L. (2011). Elucidation of extracellular matrix mechanics from muscle fibers and fiber bundles. J Biomech 44, 771-773.

Meyer, G.A. and Lieber, R.L. (in review).

Nichols, T. R. and Cope, T. C. (2004). Cross-bridge mechanisms underlying the history-dependent properties of muscle spindles and stretch reflexes. Can. J. Physiol. Pharmacol. 82, 569-576.

Prochazka, A. and Ellaway, P. (2012). Sensory systems in the control of movement. Compr Physiol 2, 2615-2627. 
Proske, U. and Stuart, G. J. (1985). The initial burst of impulses in responses of toad muscle spindles during stretch. J. Physiol. (Lond.) 368, 1-17.

Tsay, A., Savage, G., Allen, T. J. and Proske, U. (2014). Limb position sense, proprioceptive drift and muscle thixotropy at the human elbow joint. J Physiol 592, 2679-2694.

Vincent, J. A., Gabriel, H. M., Deardorff, A. S., Nardelli, P., Fyffe, R. E. W., Burkholder, T. and Cope, T. C. (2017). Muscle proprioceptors in adult rat: mechanosensory signaling and synapse distribution in spinal cord. Journal of Neurophysiology 118, 2687-2701. 